UNIVERSITÀ DEGLI STUDI DI BERGAMO

DIPARTIMENTO DI INGEGNERIA

QUADERNI DEL DIPARTIMENTO

Department of Engineering

Working Paper

Series "Mathematics and Statistics"

n. $07 / \mathrm{MS}-2013$

EQUICONVERGENCE THEOREMS FOR STURM LIOUVILLE EXPANSIONS AND SETS OF DIVERGENCE FOR BOCHNER RIESZ MEANS IN SOBOLEV SPACES

by

L. Colzani, G. Gigante, S. Volpi 
COMITATO DI REDAZIONE ${ }^{\S}$

Series Mathematics and Statistics (MS): Luca Brandolini, Alessandro Fassò, Christian Vergara

\& L'accesso alle Series è approvato dal Comitato di Redazione. I Working Papers della Collana dei Quaderni del Dipartimento di Ingegneria dell'Informazione e Metodi Matematici costituiscono un servizio atto a fornire la tempestiva divulgazione dei risultati dell'attività di ricerca, siano essi in forma provvisoria o definitiva. 


\title{
Equiconvergence theorems for Sturm Liouville expansions and sets of divergence for Bochner Riesz means in Sobolev spaces
}

\author{
Leonardo Colzani, Giacomo Gigante, and Sara Volpi
}

\begin{abstract}
We state some equiconvergence results between Bochner Riesz means of expansions in eigenfunctions of suitable Sturm Liouville operators. Then we determine the Hausdorff dimension of the divergence set of Bochner Riesz means of radial functions in Sobolev classes on Euclidean and non Euclidean spaces.
\end{abstract}

\section{Introduction}

The motivation for this paper is the study of the pointwise convergence of Bochner Riesz means of functions with some smoothness. These means are defined for suitable functions by the Fourier integrals

$$
S_{R}^{\beta} f(x)=\int_{\{|\xi|<R\}}\left(1-\left|R^{-1} \xi\right|^{2}\right)^{\beta} \widehat{f}(\xi) \exp (2 \pi i \xi x) d \xi .
$$

A classical result in this setting is the following:

Bochner Riesz means with index $\beta>(d-1)|1 / p-1 / 2|$ of functions in $\mathbb{L}^{p}\left(\mathbb{R}^{d}\right)$ converge in norm and almost everywhere: $\lim _{R \rightarrow+\infty}\left\{S_{R}^{\beta} f(x)\right\}=f(x)$.

The cases $p=1,2,+\infty$ are due to Bochner and the general case $1 \leq p \leq+\infty$ is due to Stein. See [26, Chapter VII.5]. See also $[\mathbf{5 , 6 , ~ 1 2 , ~ 2 4 ] , ~ f o r ~ b e t t e r ~ r e s u l t s ~}$ when $p>2$, and [27] for the case $p<2$. For radial functions more precise results are known:

Bochner Riesz means with index $\beta>\max \{d|1 / p-1 / 2|-1 / 2,0\}$ of radial functions in $\mathbb{L}^{p}\left(\mathbb{R}^{d}\right)$ converge in norm and almost everywhere.

See $[\mathbf{1 8}, \mathbf{2 2}]$ and $[\mathbf{2 0}]$ for extensions to symmetric spaces. For end point results in Lorentz spaces see also $[\mathbf{1 1}, \mathbf{1 4}, \mathbf{1 5}, \mathbf{2 3}]$. Other classical results on convergence of Fourier series of functions with some smoothness are due to Beurling, and Salem and Zygmund:

2010 Mathematics Subject Classification. Primary 43A55, 42B08; Secondary 43A62, 46 E35.

Key words and phrases. Equiconvergence, Sturm Liouville expansions, Bochner Riesz means, Hausdorff dimension. 
If $\sum n^{2 \gamma}\left(|a(n)|^{2}+|b(n)|^{2}\right)<+\infty$, then the set of points of divergence of the series $\sum a(n) \cos (n x)+b(n) \sin (n x)$ has outer $(1-2 \gamma)$ capacity zero if $0<\gamma<$ $1 / 2$, and it has outer logarithmic capacity zero if $\gamma=1 / 2$.

See [30, Chapter XIII.11]. A consequence of this result is that the sets of singularities of functions in Sobolev classes have small Hausdorff dimension. These results have been extended to several dimensions. In particular, it has been proved in $[\mathbf{7}, \mathbf{1 9}]$ that by putting some smoothness on the function one may decrease the index of almost everywhere summability. In [21] there is a study of the capacity and Hausdorff dimension of the divergence set of spherical partial sums of Fourier integrals. In $[\mathbf{8}, \mathbf{9}, \mathbf{1 0}]$ there is a study of the dimension of the set where the localization of spherical means fails. The related problem of the dimension of the set where a solution to a Schrödinger equation does not converge to the boundary data is considered in [1]. Finally, [13] contains the following result:

Bochner Riesz means with index $\beta>\max \{(d-1) / 2-\gamma, 0\}$ of functions with $\gamma$ derivatives in $\mathbb{L}^{1}\left(\mathbb{R}^{d}\right)$ converge pointwise, with possible exception of sets of points with Hausdorff dimension at most $d-\gamma$. Similarly, Bochner Riesz means with index $\beta \geq 0$ of functions with $\gamma$ derivatives in $\mathbb{L}^{2}\left(\mathbb{R}^{d}\right)$ converge pointwise, with possible exception of sets of points with Hausdorff dimension at most $d-2 \gamma$.

We do not know a precise analogue of the above results for functions with derivatives in $\mathbb{L}^{p}\left(\mathbb{R}^{d}\right)$ with $1 \leq p \leq+\infty$, but at least for radial functions there are some definitive results. In particular, here we want to prove the following:

Let $\beta \geq 0, \gamma \geq 0,1 \leq p<+\infty,(d-1-2 \beta) / 2 d<1 / p<(d+1+2 \beta+2 \gamma) / 2 d$. Then the Bochner Riesz means with index $\beta$ of radial functions with $\gamma$ derivatives in $\mathbb{L}^{p}\left(\mathbb{R}^{d}\right)$ converge pointwise, with possible exception of sets of points $\Omega$ with the following properties:

(1) if $\gamma p \leq 1$, then the Hausdorff dimension of $\Omega$ is at most $d-\gamma p$.

(2) if $1<\gamma p \leq d$, then $\Omega$ either is empty or it reduces to the origin.

(3) if $\gamma p>d$, then $\Omega$ is empty.

Divergence of Bochner Riesz means of radial functions occurs in spheres $\{|x|=r\}$, and sets of spheres of dimension $d-\gamma p$ in $\mathbb{R}^{d}$ correspond to sets of radii of dimension $1-\gamma p$ in $\mathbb{R}_{+}$. Observe that the above results are best possible, since functions with $\gamma$ derivatives in $\mathbb{L}^{p}\left(\mathbb{R}^{d}\right)$ can be infinite on sets with dimension $d-\gamma p$. Also observe the asymmetry between $p<2$ and $p>2$. When $p<2$ if the smoothness index $\gamma$ increases then the critical index $\beta=d(1 / p-1 / 2)-1 / 2-\gamma$ for summability decreases, but when $p>2$ the critical index $\beta=d(1 / 2-1 / p)-1 / 2$ for summability is independent of the smoothness. Indeed it is even possible to prove more precise results for Lorentz spaces and for expansions in eigenfunctions of Sturm Liouville problems, such as Fourier Bessel expansions.

The plan of the paper is the following: The first section contains an equiconvergence result between Bochner Riesz means of trigonometric and Bessel expansions: Under appropriate integrability assumptions, the Bochner Riesz means of the Fourier Bessel expansion of a given function converge at a given point if and only if the means associated to the Fourier cosine expansion converge at the point. In particular, this implies equiconvergence between Bochner Riesz 
means of radial functions in one and several dimensions. The second section generalizes the first, since it contains an equiconvergence result between Bochner Riesz means of Bessel expansions and expansions in eigenfunctions of Sturm Liouville operators $-A(x)^{-1} \frac{d}{d x}\left(A(x) \frac{d}{d x}\right)$. In particular, when $A(x)=x^{d-1}$ then this operator is the radial component of the Laplacian in $\mathbb{R}^{d}$, and when $A(x)=$ $\sinh ^{2 \alpha+1}(x) \cosh ^{2 \alpha^{\prime}+1}(x)$ with suitable $\alpha$ and $\alpha^{\prime}$ then it is the radial component of the Laplace Beltrami operator on non compact rank one symmetric spaces. In the third section these equiconvergence results are applied to functions in Sobolev classes.

\section{Equiconvergence between trigonometric and Bessel expansions}

Trigonometric expansions of radial functions in $\mathbb{R}^{d}$ are particular cases of Fourier Bessel expansions, and in the sequel we shall deal with this slightly more general context. The Bessel functions $(t x)^{-\alpha} J_{\alpha}(t x)$ are analytic eigenfunctions of the radial component of the Laplace operator,

$$
-\left(\frac{d^{2}}{d x^{2}}+\frac{2 \alpha+1}{x} \frac{d}{d x}\right) \frac{J_{\alpha}(t x)}{(t x)^{\alpha}}=t^{2} \frac{J_{\alpha}(t x)}{(t x)^{\alpha}} .
$$

For $\alpha \geq-1 / 2$ the Fourier Bessel transform and its inversion formula are

$$
\mathcal{F}_{\alpha} f(t)=\int_{0}^{+\infty} f(y) \frac{J_{\alpha}(t y)}{(t y)^{\alpha}} y^{2 \alpha+1} d y, \quad f(x)=\int_{0}^{+\infty} \mathcal{F}_{\alpha} f(t) \frac{J_{\alpha}(t x)}{(t x)^{\alpha}} t^{2 \alpha+1} d t .
$$

The parameter $2 \alpha+2$ plays the role of space dimension and when $\alpha=-1 / 2$, then $J_{-1 / 2}(z)=\sqrt{2 / \pi z} \cos (z)$ and the Fourier Bessel transform reduces to the cosine transform,

$$
\mathcal{F}_{-1 / 2} f(t)=\sqrt{\frac{2}{\pi}} \int_{0}^{+\infty} f(y) \cos (t y) d y, \quad f(x)=\sqrt{\frac{2}{\pi}} \int_{0}^{+\infty} \mathcal{F}_{-1 / 2} f(t) \cos (t x) d t .
$$

The Bochner Riesz means of Fourier Bessel expansions of order $\beta \geq 0$ are defined as

$$
\begin{gathered}
S_{R}^{\beta} f(x)=\int_{0}^{R}\left(1-(t / R)^{2}\right)^{\beta} \mathcal{F}_{\alpha} f(t) \frac{J_{\alpha}(t x)}{(t x)^{\alpha}} t^{2 \alpha+1} d t \\
=\int_{0}^{+\infty}\left(\int_{0}^{R}\left(1-(t / R)^{2}\right)^{\beta} \frac{J_{\alpha}(t x)}{(t x)^{\alpha}} \frac{J_{\alpha}(t y)}{(t y)^{\alpha}}(t y)^{2 \alpha+1} d t\right) f(y) d y .
\end{gathered}
$$

Bessel functions have simple asymptotic expansions in terms of trigonometric functions. Using these asymptotic expansions, we shall prove that the means $S_{R}^{\beta} f(x)$ are equiconvergent with the Bochner Riesz means of the cosine expansion of $f(x)$. Let $0<\varepsilon<\eta<+\infty$ and let $\chi(x)$ be a smooth cut off with $\chi(x)=1$ if $\varepsilon / 2<x<2 \eta$ and $\chi(x)=0$ if $x<\varepsilon / 3$ or $x>3 \eta$. Define

$$
\begin{aligned}
& T_{R}^{\beta} f(x)=\sqrt{2 / \pi} \int_{0}^{R}\left(1-(t / R)^{2}\right)^{\beta} \mathcal{F}_{-1 / 2}(\chi f)(t) \cos (t x) d t \\
= & \int_{0}^{+\infty}\left((2 / \pi) \chi(y) \int_{0}^{R}\left(1-(t / R)^{2}\right)^{\beta} \cos (t x) \cos (t y) d t\right) f(y) d y .
\end{aligned}
$$

The following is an equiconvergence result between $S_{R}^{\beta} f(x)$ and $T_{R}^{\beta} f(x)$. 
Theorem 1. Let $\alpha \geq-1 / 2, \beta \geq 0, \lambda=\min \{\alpha+1 / 2, \beta\}$, and assume that

$$
\int_{0}^{+\infty}|f(x)| \frac{x^{\alpha+\lambda+1 / 2}}{(1+x)^{\beta+\lambda+1}} d x<+\infty .
$$

Then the means $S_{R}^{\beta} f(x)$ and $T_{R}^{\beta} f(x)$ are uniformly equiconvergent in $0<\varepsilon<x<$ $\eta<+\infty$ as $R \rightarrow+\infty$, that is

$$
\lim _{R \rightarrow+\infty}\left\{\sup _{\varepsilon<x<\eta}\left|S_{R}^{\beta} f(x)-T_{R}^{\beta} f(x)\right|\right\}=0 .
$$

Proof. The case $\beta=0$ is already in $[\mathbf{1 4}]$ and the case $\beta>0$ is implicitly but essentially contained in $[\mathbf{1 5}]$. The idea is that the main term in the asymptotic expansion of the kernel associated to the operator $S_{R}^{\beta}$ is independent of $\alpha$ and it coincides with the kernel of the operator $T_{R}^{\beta}$ and, under appropriate assumptions, the contribution of the remainder in the asymptotic expansion of the kernel is negligible. Write

$$
\begin{aligned}
& S_{R}^{\beta} f(x)=\int_{0}^{+\infty} S_{R}^{\beta}(x, y) f(y) d y, \\
& T_{R}^{\beta} f(x)=\int_{0}^{+\infty} T_{R}^{\beta}(x, y) f(y) d y,
\end{aligned}
$$

with the kernels $S_{R}^{\beta}(x, y)$ and $T_{R}^{\beta}(x, y)$ defined by

$$
\begin{gathered}
S_{R}^{\beta}(x, y)=\int_{0}^{R}\left(1-(t / R)^{2}\right)^{\beta} \frac{J_{\alpha}(t x)}{(t x)^{\alpha}} \frac{J_{\alpha}(t y)}{(t y)^{\alpha}}(t y)^{2 \alpha+1} d t, \\
T_{R}^{\beta}(x, y)=(2 / \pi) \chi(y) \int_{0}^{R}\left(1-(t / R)^{2}\right)^{\beta} \cos (t x) \cos (t y) d t .
\end{gathered}
$$

Now, for every function $f(x)$ and $g(x)$,

$$
\left|S_{R}^{\beta} f(x)-T_{R}^{\beta} f(x)\right| \leq\left|\left(S_{R}^{\beta}-T_{R}^{\beta}\right)(f-g)(x)\right|+\left|\left(S_{R}^{\beta}-T_{R}^{\beta}\right) g(x)\right| .
$$

Then the theorem follows from the following claims:

(1) If $g(x)$ is a smooth function with compact support and if $\varepsilon<x<\eta$, then

$$
\lim _{R \rightarrow+\infty}\left\{S_{R}^{\beta} g(x)\right\}=\lim _{R \rightarrow+\infty}\left\{T_{R}^{\beta} g(x)\right\}=g(x) .
$$

(2) If $\varepsilon<x<\eta$, then

$$
\left|S_{R}^{\beta}(x, y)-T_{R}^{\beta}(x, y)\right| \leq c \frac{y^{\alpha+\lambda+1 / 2}}{(1+y)^{\beta+\lambda+1}} .
$$

The first claim is the classical Fourier inversion formula for smooth functions. For a short and elementary proof see $[\mathbf{1 4}]$. The proof of the second claim is contained in the following lemmas, and it is finished after Lemma 3. 
Lemma 1. The kernel of the operator $T_{R}^{\beta}$ is

$$
\begin{gathered}
T_{R}^{\beta}(x, y)=\pi^{-1} \chi(y) \int_{0}^{R}\left(1-(t / R)^{2}\right)^{\beta} \cos (t(x-y)) d t \\
+\pi^{-1} \chi(y) \int_{0}^{R}\left(1-(t / R)^{2}\right)^{\beta} \cos (t(x+y)) d t \\
=\chi(y) \frac{2^{\beta} \Gamma(\beta+1)}{\sqrt{2 \pi}} R\left(\frac{J_{\beta+1 / 2}(R|x-y|)}{(R|x-y|)^{\beta+1 / 2}}+\frac{J_{\beta+1 / 2}(R|x+y|)}{(R|x+y|)^{\beta+1 / 2}}\right) .
\end{gathered}
$$

Proof. This follows from the integral representation of Bessel functions:

$$
J_{\beta+1 / 2}(z)=2^{1 / 2-\beta} \pi^{-1 / 2} \Gamma(\beta+1)^{-1} z^{\beta+1 / 2} \int_{0}^{1}\left(1-t^{2}\right)^{\beta} \cos (z t) d t .
$$

Lemma 2. The kernel of the operator $S_{R}^{\beta}$ is

$$
S_{R}^{\beta}(x, y)=\int_{0}^{R}\left(1-(t / R)^{2}\right)^{\beta} \frac{J_{\alpha}(t x)}{(t x)^{\alpha}} \frac{J_{\alpha}(t y)}{(t y)^{\alpha}}(t y)^{2 \alpha+1} d t .
$$

This kernel satisfies the estimates:

$$
\begin{gathered}
\text { (1) }\left|S_{R}^{\beta}(x, y)\right| \leq c R^{2 \alpha+2} y^{2 \alpha+1}(1+R|x-y|)^{-\alpha-\beta-3 / 2}, \\
\text { (2) }\left|S_{R}^{\beta}(x, y)\right| \leq c R^{-\beta} x^{-\alpha-\beta-3 / 2} y^{\alpha+1 / 2} \quad \text { if } 2 y<x, \\
\text { (3) }\left|S_{R}^{\beta}(x, y)\right| \leq c R^{-\beta} x^{-\alpha-1 / 2} y^{\alpha-\beta-1 / 2} \quad \text { if } 2 x<y, \\
\text { (4) }\left|S_{R}^{\beta}(x, y)-\frac{2^{\beta} \Gamma(\beta+1)}{\sqrt{2 \pi}} R \frac{J_{\beta+1 / 2}(R|x-y|)}{(R|x-y|)^{\beta+1 / 2}}\right| \leq c \\
\text { if } 0<\varepsilon<x, y<\eta<+\infty .
\end{gathered}
$$

Proof. This has been proved in [15]. Here we just hint at the proof of (4), which is the main ingredient in what follows. The asymptotic expansion of Bessel functions is

$$
J_{\alpha}(z)=\sqrt{2 / \pi z} \cos (z-\alpha \pi / 2-\pi / 4)+\ldots .
$$

Hence the asymptotic expansion of the kernel $S_{R}^{\beta}(x, y)$ is

$$
\begin{aligned}
& \int_{0}^{R}\left(1-(t / R)^{2}\right)^{\beta} \frac{J_{\alpha}(t x)}{(t x)^{\alpha}} \frac{J_{\alpha}(t y)}{(t y)^{\alpha}}(t y)^{2 \alpha+1} d t \\
= & x^{-\alpha} y^{\alpha+1} R^{2} \int_{0}^{1} s\left(1-s^{2}\right)^{\beta} J_{\alpha}(R x s) J_{\alpha}(R y s) d s \\
= & \pi^{-1}(y / x)^{\alpha+1 / 2} R \int_{0}^{1}\left(1-s^{2}\right)^{\beta} \cos (R(x-y) s) d s \\
+\pi^{-1} & (y / x)^{\alpha+1 / 2} R \int_{0}^{1}\left(1-s^{2}\right)^{\beta} \sin (R(x+y) s-\alpha \pi) d s+\ldots \\
= & \frac{2^{\beta} \Gamma(\beta+1)}{\sqrt{2 \pi}}\left(\frac{y}{x}\right)^{\alpha+1 / 2} R \frac{J_{\beta+1 / 2}(R|x-y|)}{(R|x-y|)^{\beta+1 / 2}}+\ldots
\end{aligned}
$$


Indeed, when $\alpha=n+1 / 2$ is a half integer, the asymptotic expansions of $J_{\alpha}(z)$ and $S_{R}^{\beta}(x, y)$ are finite equalities, and the desired estimates are easily verified. The terms with $x+y$ are more oscillating than the ones with $x-y$, hence they are less singular. Finally, when $\varepsilon<x, y<\eta$ one can get rid of the factor $(y / x)^{\alpha+1 / 2}$,

$$
\begin{gathered}
\left|\left(\frac{y}{x}\right)^{\alpha+1 / 2} R \frac{J_{\beta+1 / 2}(R|x-y|)}{(R|x-y|)^{\beta+1 / 2}}-R \frac{J_{\beta+1 / 2}(R|x-y|)}{(R|x-y|)^{\beta+1 / 2}}\right| \\
=R\left|(y / x)^{\alpha+1 / 2}-1\right|\left|(R|x-y|)^{-\beta-1 / 2} J_{\beta+1 / 2}(R|x-y|)\right| \\
\leq c(R|x-y|)^{-\beta+1 / 2}\left|J_{\beta+1 / 2}(R|x-y|)\right| \\
\leq \begin{cases}c(R|x-y|) & \text { if } R|x-y| \leq 1, \\
c(R|x-y|)^{-\beta} & \text { if } R|x-y| \geq 1 .\end{cases}
\end{gathered}
$$

LEMma 3. There exists a constant $c$ such that for every $\varepsilon<x<\eta$,

$$
\left|S_{R}^{\beta}(x, y)-T_{R}^{\beta}(x, y)\right| \leq c \frac{y^{\alpha+\lambda+1 / 2}}{(1+y)^{\beta+\lambda+1}} .
$$

Proof. We may assume $R>3 / \varepsilon$. If $0<y<1 / R$ then, by Lemma $2(1)$,

$$
\left|S_{R}^{\beta}(x, y)-T_{R}^{\beta}(x, y)\right|=\left|S_{R}^{\beta}(x, y)\right| \leq c R^{\alpha-\beta+1 / 2} y^{2 \alpha+1} \leq c y^{\alpha+\lambda+1 / 2} .
$$

If $1 / R<y<\varepsilon / 3$ then, by Lemma $2(2)$,

$$
\left|S_{R}^{\beta}(x, y)-T_{R}^{\beta}(x, y)\right|=\left|S_{R}^{\beta}(x, y)\right| \leq c R^{-\beta} y^{\alpha+1 / 2} \leq c y^{\alpha+\lambda+1 / 2} .
$$

If $\varepsilon / 3<y<\varepsilon / 2$ then, by Lemma 1 and Lemma $2(2)$, and the estimate $\left|J_{\beta+1 / 2}(z)\right| \leq$ $c z^{-1 / 2}$,

$$
\left|S_{R}^{\beta}(x, y)-T_{R}^{\beta}(x, y)\right| \leq\left|S_{R}^{\beta}(x, y)\right|+\left|T_{R}^{\beta}(x, y)\right| \leq c R^{-\beta} .
$$

If $\varepsilon / 2<y<2 \eta$ then, by Lemma 1 and Lemma 2 (4),

$$
\left|S_{R}^{\beta}(x, y)-T_{R}^{\beta}(x, y)\right| \leq c .
$$

If $2 \eta<y<3 \eta$ then, by Lemma 1 and Lemma $2(3)$,

$$
\left|S_{R}^{\beta}(x, y)-T_{R}^{\beta}(x, y)\right| \leq\left|S_{R}^{\beta}(x, y)\right|+\left|T_{R}^{\beta}(x, y)\right| \leq c R^{-\beta} .
$$

Finally, if $y>3 \eta$ then, by Lemma $2(3)$,

$$
\left|S_{R}^{\beta}(x, y)-T_{R}^{\beta}(x, y)\right|=\left|S_{R}^{\beta}(x, y)\right| \leq c R^{-\beta} y^{\alpha-\beta-1 / 2} .
$$

Corollary 1. Let $1 \leq p \leq+\infty$ and $(2 \alpha-2 \beta+1) /(4 \alpha+4)<1 / p<$ $(2 \alpha+2 \beta+3) /(4 \alpha+4)$. Then for every function $f(x)$ in $L^{p}\left(\mathbb{R}_{+}, x^{2 \alpha+1} d x\right)$ the means $S_{R}^{\beta} f(x)$ and $T_{R}^{\beta} f(x)$ are equiconvergent in $\varepsilon<x<\eta$. 
Proof. It suffices to show that the hypotheses in Theorem 1 are satisfied. Indeed, by Hölder inequality with $1 / p+1 / q=1$,

$$
\begin{gathered}
\int_{0}^{+\infty}|f(x)| \frac{x^{\alpha+\lambda+1 / 2}}{(1+x)^{\beta+\lambda+1}} d x \\
\leq\left\{\int_{0}^{+\infty}|f(x)|^{p} x^{2 \alpha+1} d x\right\}^{1 / p}\left\{\int_{0}^{+\infty}\left|\frac{x^{\lambda-\alpha-1 / 2}}{(1+x)^{\beta+\lambda+1}}\right|^{q} x^{2 \alpha+1} d x\right\}^{1 / q} .
\end{gathered}
$$

The second integral is easily seen to be finite for the given set of parameters.

REMARK 1. The factor $R^{-\beta}$ in the proof of Lemma 3 suggests the possibility of an improvement. Indeed, when $\beta>0$ then equiconvergence holds also at the lower critical index $p=(4 \alpha+4) /(2 \alpha+2 \beta+3)$. On the other hand, when $p<$ $(4 \alpha+4) /(2 \alpha+2 \beta+3)$ there exist functions in $\mathbb{L}^{p}\left(\mathbb{R}_{+}, x^{2 \alpha+1} d x\right)$ with Bochner Riesz means of order $\beta$ diverging everywhere, and when $p \geq(4 \alpha+4) /(2 \alpha-2 \beta+1)$ these means are not even defined as tempered distributions. Finally, more precise results hold when the size of functions is measured by Lorentz norms. When $\beta>0$ and $p=(4 \alpha+4) /(2 \alpha+2 \beta+3)$ then equiconvergence holds for functions in the closure of test functions in $\mathbb{L}^{p, \infty}\left(\mathbb{R}_{+}, x^{2 \alpha+1} d x\right)$, and when $p=(4 \alpha+4) /(2 \alpha-2 \beta+1)$ then equiconvergence holds in $\mathbb{L}^{p, 1}\left(\mathbb{R}_{+}, x^{2 \alpha+1} d x\right)$. See $[\mathbf{1 4}, \mathbf{1 5}, \mathbf{2 8}]$, and the remarks in the following sections.

\section{Sturm Liouville expansions}

In this section we consider expansions in eigenfunctions of Sturm Liouville operators on $0<x<+\infty$ of the form

$$
\mathcal{L}=-A^{-1}(x) \frac{d}{d x}\left(A(x) \frac{d}{d x}\right) .
$$

This operator is formally self adjoint with respect to the measure $A(x) d x$. For example, when $A(x)=x^{d-1}$ then $\mathcal{L}$ is the radial component of the Laplacian in $\mathbb{R}^{d}$, when $A(x)=x^{2 \alpha+1}$ then $\mathcal{L}$ is the Bessel operator of the previous section, and when $A(x)=\sinh ^{2 \alpha+1}(x) \cosh ^{2 \alpha^{\prime}+1}(x)$ with suitable $\alpha$ and $\alpha^{\prime}$ then $\mathcal{L}$ is the radial component of the Laplace Beltrami operator on non compact rank one symmetric spaces. In what follows we assume that the operator $\mathcal{L}$ is a perturbation of the Bessel operator. More precisely, as in [4] we assume the following:

(1) $A(x)$ is continuous in $0 \leq x<+\infty$, positive non decreasing and smooth in $0<x<+\infty$, and $\lim _{x \rightarrow+\infty} A(x)=+\infty$.

(2) $A^{\prime}(x) / A(x)$ is decreasing in $0<x<+\infty$ and there exists $\alpha>-1 / 2$ and a smooth odd function $B(x)$ such that

$$
\frac{A^{\prime}(x)}{A(x)}=\frac{2 \alpha+1}{x}+B(x)
$$

In particular, $A(x) \approx c x^{2 \alpha+1}$ as $x \rightarrow 0+$, and with a change of variable one can assume that $c=1$. Set $\lim _{x \rightarrow+\infty} A^{\prime}(x) / A(x)=2 \rho$. For every $t \in \mathbb{C}$ the Cauchy problem

$$
\left\{\begin{array}{l}
\mathcal{L} u(x)=\left(t^{2}+\rho^{2}\right) u(x), \\
u(0)=1, \quad u^{\prime}(0)=0
\end{array}\right.
$$


has a unique solution $\varphi_{t}(x)$ defined in $0 \leq x<+\infty$, and if $t$ is real then $\left|\varphi_{t}(x)\right| \leq 1$. Moreover, there exists a function $c(t)$, the Harish-Chandra function, such that for every test function one can define the Fourier transform and an inversion formula

$$
\mathcal{F} f(t)=\int_{0}^{+\infty} f(y) \varphi_{t}(y) A(y) d y, \quad f(x)=\int_{0}^{+\infty} \mathcal{F} f(t) \varphi_{t}(x) \frac{d t}{2 \pi|c(t)|^{2}}
$$

By means of the Liouville transformation $\sqrt{A(x)} u(x)=v(x)$, the equation $\mathcal{L} u(x)=\left(t^{2}+\rho^{2}\right) u(x)$ becomes

$$
\left(\frac{d^{2}}{d x^{2}}+t^{2}\right) v(x)=q(x) v(x)
$$

with

$$
q(x)=\frac{1}{2} \frac{d}{d x}\left(\frac{A^{\prime}(x)}{A(x)}\right)+\frac{1}{4}\left(\frac{A^{\prime}(x)}{A(x)}\right)^{2}-\rho^{2} .
$$

We also assume the following:

(3) There exists $a \geq 0$ such that when $x \rightarrow+\infty$,

$$
q(x)=\frac{a^{2}-1 / 4}{x^{2}}+\zeta(x)
$$

with $\int_{1}^{+\infty}|\zeta(x)| x \log (x) d x<+\infty$ if $a=0$, or $\int_{1}^{+\infty}|\zeta(x)| x d x<+\infty$ if $a>0$.

Under these assumptions, it is proved in [4] that when $a>0$ then $\sqrt{A(x)} \varphi_{0}(x) \approx$ $c x^{1 / 2+b}$ as $x \rightarrow+\infty$, for some $b>-1 / 2$ and $|b|=a$. On the other hand, when $a=0$ then $\sqrt{A(x)} \varphi_{0}(x) \approx c x^{1 / 2}$ or $c x^{1 / 2} \log (x)$ as $x \rightarrow+\infty$, and in this case we set $b=0$. Observe that if $\rho=0$ then $\varphi_{0}(x)=1$.

Finally, we also assume the following:

(4) If $-1 / 2<b<0$, then

$$
\int_{1}^{+\infty}|\zeta(x)| x^{2|b|+1} d x<+\infty .
$$

If $b=0$ and $\sqrt{A(x)} \varphi_{0}(x) \approx c x^{1 / 2}$ as $x \rightarrow+\infty$, then

$$
\int_{1}^{+\infty}|\zeta(x)| x \log ^{2}(x) d x<+\infty \text {. }
$$

This last assumption $b \leq 0$ occurs only if $\rho=0$. It turns out that, as in the case of Bessel expansions, the constant $2 \alpha+2$ plays the role of the dimensions of the space at 0 , while when $\rho=0$, then $2 b+2$ plays the role of the dimensions of the space at $+\infty$.

As we said, an explicit example relevant to the harmonic analysis on hyperbolic spaces is $A(x)=\sinh ^{2 \alpha+1}(x) \cosh ^{2 \alpha^{\prime}+1}(x)$. In this case $\varphi_{t}(x)$ is a Jacobi function,

$$
\varphi_{t}(x)=F\left(\left(\alpha+\alpha^{\prime}+1-i t\right) / 2,\left(\alpha+\alpha^{\prime}+1+i t\right) / 2 ; \alpha+1 ;-\sinh ^{2}(t)\right),
$$

and $c(t)$ is the Harish-Chandra function

$$
c(t)=\frac{\Gamma(\alpha+1) \Gamma(i t / 2) \Gamma((1+i t) / 2)}{2 \sqrt{\pi} \Gamma\left(\left(\alpha-\alpha^{\prime}+1+i t\right) / 2\right) \Gamma\left(\left(\alpha+\alpha^{\prime}+1+i t\right) / 2\right)} .
$$

One can easily check that $A^{\prime}(x) / A(x)$ is decreasing if and only if $\alpha \geq \alpha^{\prime}$, and

$$
2 \rho=\lim _{x \rightarrow+\infty} \frac{A^{\prime}(x)}{A(x)}=\lim _{x \rightarrow+\infty} \frac{\left(2 \alpha+2 \alpha^{\prime}+2\right) \cosh ^{2}(x)-2 \alpha^{\prime}-1}{\sinh (x) \cosh (x)}=2 \alpha+2 \alpha^{\prime}+2 .
$$


The Bochner Riesz means with index $\beta$ of Sturm Liouville expansions are

$$
\begin{gathered}
W_{R}^{\beta} f(x)=\int_{0}^{R}\left(1-(t / R)^{2}\right)^{\beta} \mathcal{F} f(t) \varphi_{t}(x) \frac{d t}{2 \pi|c(t)|^{2}} \\
=\int_{0}^{+\infty}\left(A(y) \int_{0}^{R}\left(1-(t / R)^{2}\right)^{\beta} \varphi_{t}(x) \varphi_{t}(y) \frac{d t}{2 \pi|c(t)|^{2}}\right) f(y) d y .
\end{gathered}
$$

As we said, $\mathcal{L}$ is a perturbation of the Bessel operator, and the eigenfunctions $\varphi_{t}(x)$ have asymptotic expansions in terms of Bessel functions. This suggests the possibility of equiconvergence between these Sturm Liouville and cosine expansions $W_{R}^{\beta} f(x)$ and $T_{R}^{\beta} f(x)$ defined in the previous section.

THEOREM 2. Let $A(x)$ satisfy the above conditions (1) through (4) with $\alpha>$ $-1 / 2$, and let $\beta \geq 0$ and $\lambda=\min \{\alpha+1 / 2, \beta\}$. Finally, assume that

$$
\int_{0}^{+\infty}|f(x)| \frac{\sqrt{A(x)} x^{\lambda}}{(1+x)^{\lambda+\beta+1}} d x<+\infty .
$$

Then the means $W_{R}^{\beta} f(x)$ and $T_{R}^{\beta} f(x)$ are uniformly equiconvergent in $0<\varepsilon<x<$ $\eta<+\infty$ as $R \rightarrow+\infty$, that is

$$
\lim _{R \rightarrow+\infty} \sup _{\varepsilon<x<\eta}\left\{\left|W_{R}^{\beta} f(x)-T_{R}^{\beta} f(x)\right|\right\}=0 .
$$

Proof. The case $\beta=0$ of this theorem is already in [4]. The proof of the case $\beta>0$ is similar. With the notation of the previous section, define

$$
\begin{gathered}
V_{R}^{\beta} f(x)=\sqrt{x^{2 \alpha+1} / A(x)} S_{R}^{\beta}\left(\sqrt{A(y) / y^{2 \alpha+1}} f(y)\right)(x) \\
=\int_{0}^{+\infty}\left(\sqrt{A(y) / A(x)} \int_{0}^{R}\left(1-(t / R)^{2}\right)^{\beta} \sqrt{t x} J_{\alpha}(t x) \sqrt{t y} J_{\alpha}(t y) d t\right) f(y) d y .
\end{gathered}
$$

It suffices to show that the Sturm Liouville expansions $W_{R}^{\beta} f(x)$ are equiconvergent with the Bessel expansions $V_{R}^{\beta} f(x)$, and that these Bessel expansions $V_{R}^{\beta} f(x)$ are equiconvergent with the trigonometric expansions $T_{R}^{\beta} f(x)$. This is done in the following lemmas.

LEMMA 4. (1) Let $V_{R}^{\beta}(x, y)$ and $W_{R}^{\beta}(x, y)$ be the kernels of the operators $V_{R}^{\beta}$ and $W_{R}^{\beta}$,

$$
\begin{gathered}
V_{R}^{\beta}(x, y)=\sqrt{A(y) / A(x)} \int_{0}^{R}\left(1-(t / R)^{2}\right)^{\beta} \sqrt{t x} J_{\alpha}(t x) \sqrt{t y} J_{\alpha}(t y) d t \\
W_{R}^{\beta}(x, y)=A(y) \int_{0}^{R}\left(1-(t / R)^{2}\right)^{\beta} \varphi_{t}(x) \varphi_{t}(y) \frac{d t}{2 \pi|c(t)|^{2}} .
\end{gathered}
$$

Then for every $0<x<+\infty$ there exists a constant $c$ such that

(2) Assume that

$$
\left|V_{R}^{\beta}(x, y)-W_{R}^{\beta}(x, y)\right| \leq c \frac{\sqrt{A(y)} y^{\lambda}}{(1+y)^{\lambda+\beta+1}} .
$$

$$
\int_{0}^{+\infty}|f(x)| \frac{\sqrt{A(x)} x^{\lambda}}{(1+x)^{\lambda+\beta+1}} d x<+\infty .
$$


Then for every $0<x<+\infty$,

$$
\lim _{R \rightarrow+\infty}\left\{\left|W_{R}^{\beta} f(x)-V_{R}^{\beta} f(x)\right|\right\}=0 .
$$

Moreover, in (1) one can choose the same constant for all $\varepsilon<x<\eta$ and in (2) the convergence is uniform in $\varepsilon<x<\eta$.

Proof. The following proof relies heavily on [4]. As it is well known, for every $t \in \mathbb{C}-\{0\}$ the differential equation

$$
\mathcal{L} u(x)=\left(t^{2}+\rho^{2}\right) u(x)
$$

has a unique solution $\Phi_{t}(x)$ over $0<x<+\infty$ twice continuously differentiable and satisfying the condition at infinity

$$
\sqrt{A(x)} \Phi_{t}(x)=e^{i t x}(1+\mathcal{R}(t, x)),
$$

with $\mathcal{R}(t, x) \rightarrow 0$ and $\partial \mathcal{R}(t, x) / \partial x \rightarrow 0$ as $t \rightarrow+\infty$. For any $t$ in $\mathbb{C}-\{0\}, \Phi_{t}(x)$ and $\Phi_{-t}(x)$ are two independent solutions of $\mathcal{L} u(x)=\left(t^{2}+\rho^{2}\right) u(x)$, and the Harish-Chandra function is defined precisely as the coefficient $c(t)$ that realizes the identity

$$
\varphi_{t}(x)=c(t) \Phi_{t}(x)+c(-t) \Phi_{-t}(x) .
$$

Assume $0<y<x$. If $t$ is real, then $c(-t)=\overline{c(t)}$. Hence,

$$
\begin{gathered}
W_{R}^{\beta}(x, y)=A(y) \int_{0}^{R}\left(1-(t / R)^{2}\right)^{\beta} \varphi_{t}(x) \varphi_{t}(y) \frac{d t}{2 \pi|c(t)|^{2}} \\
=A(y) \int_{0}^{R}\left(1-(t / R)^{2}\right)^{\beta}\left(c(t) \Phi_{t}(x)+c(-t) \Phi_{-t}(x)\right) \varphi_{t}(y) \frac{d t}{2 \pi|c(t)|^{2}} \\
=A(y) \int_{-R}^{R}\left(1-(t / R)^{2}\right)^{\beta} \Phi_{-t}(x) \varphi_{t}(y) \frac{d t}{2 \pi c(t)} .
\end{gathered}
$$

The above formula holds also in the particular case $A(t)=t^{2 \alpha+1}$, with the functions $\Phi_{-t}(x), \varphi_{t}(y)$, and $c(t)$ replaced respectively by

$$
\begin{aligned}
\widetilde{\boldsymbol{\Phi}}_{-t}(x) & =\sqrt{\frac{\pi t}{2}} e^{i \frac{\pi}{4}(2 \alpha+1)} x^{-\alpha} H_{\alpha}^{(2)}(t x), \\
\widetilde{\boldsymbol{\varphi}}_{t}(y) & =2^{\alpha} \Gamma(\alpha+1) \frac{J_{\alpha}(t y)}{(t y)^{\alpha}} \\
\widetilde{\boldsymbol{c}}(t) & =\frac{2^{\alpha} \Gamma(\alpha+1) e^{-i \frac{\pi}{4}(2 \alpha+1)}}{\sqrt{2 \pi} t^{\alpha+\frac{1}{2}}}
\end{aligned}
$$

Here $H_{\alpha}^{(2)}(z)$ is the Bessel function of third kind, or Hankel function of order $\alpha$. Thus

$$
S_{R}^{\beta}(x, y)=y^{2 \alpha+1} \int_{-R}^{R}\left(1-(t / R)^{2}\right)^{\beta} \widetilde{\boldsymbol{\Phi}}_{-t}(x) \widetilde{\boldsymbol{\varphi}}_{t}(y) \frac{d t}{2 \pi \widetilde{\boldsymbol{c}}(t)} .
$$

Therefore

$$
V_{R}^{\beta}(x, y)=\sqrt{x^{2 \alpha+1} y^{2 \alpha+1} A(y) / A(x)} \int_{-R}^{R}\left(1-(t / R)^{2}\right)^{\beta} \widetilde{\boldsymbol{\Phi}}_{-t}(x) \widetilde{\boldsymbol{\varphi}}_{t}(y) \frac{d t}{2 \pi \widetilde{\boldsymbol{c}}(t)} .
$$


It then follows that

$$
\begin{gathered}
W_{R}^{\beta}(x, y)-V_{R}^{\beta}(x, y) \\
=\sqrt{A(y) / A(x)} \int_{-R}^{R}\left(1-(t / R)^{2}\right)^{\beta} \\
\times\left(\sqrt{A(y)} \varphi_{t}(y) \sqrt{A(x)} \frac{\Phi_{-t}(x)}{2 \pi c(t)}-\sqrt{y^{2 \alpha+1}} \widetilde{\boldsymbol{\varphi}}_{t}(y) \sqrt{x^{2 \alpha+1}} \frac{\widetilde{\boldsymbol{\Phi}}_{-t}(x)}{2 \pi \widetilde{\boldsymbol{c}}(t)}\right) d t .
\end{gathered}
$$

In our hypotheses, the eigenfunctions $\varphi_{t}(y)$ and $\widetilde{\varphi}_{t}(y)$ are entire in $t$, while $\Phi_{-t}(x) / c(t)$ and $\widetilde{\boldsymbol{\Phi}}_{-t}(x) / \widetilde{\boldsymbol{c}}(t)$ are continuous in $\{\operatorname{Im} t \leq 0\}$ and analytic in $\{\operatorname{Im} t<0\}$. See Theorems 1.19 and 2.4 in [4]. We can therefore estimate the above integral after a modification of the path of integration. If $\omega=\left\{R e^{i \theta}:-\pi \leq \theta \leq 0\right\}$, then

$$
\begin{gathered}
W_{R}^{\beta}(x, y)-V_{R}^{\beta}(x, y) \\
=\sqrt{A(y) / A(x)} \int_{\omega}\left(1-(t / R)^{2}\right)^{\beta} \\
\times\left(\sqrt{A(y)} \varphi_{t}(y) \sqrt{A(x)} \frac{\Phi_{-t}(x)}{2 \pi c(t)}-\sqrt{y^{2 \alpha+1}} \widetilde{\varphi}_{t}(y) \sqrt{x^{2 \alpha+1}} \frac{\widetilde{\boldsymbol{\Phi}}_{-t}(x)}{2 \pi \widetilde{\boldsymbol{c}}(t)}\right) d t .
\end{gathered}
$$

Now observe that, under the hypotheses (1), (2), (3) and (4) above, by [4, Theorems $1.2,1.17$ and 2.1], the following estimates hold uniformly in $|t| \geq 1, x>\varepsilon>0$ and $y>0$,

$$
\begin{gathered}
\sqrt{A(y)} \varphi_{t}(y)=\sqrt{y^{2 \alpha+1}} \widetilde{\boldsymbol{\varphi}}_{t}(y)+R_{0}(t, y), \quad\left|R_{0}(t, y)\right| \leq c|t|^{-\alpha-3 / 2} e^{|\operatorname{Im}(t y)|}, \\
\sqrt{A(x)} \Phi_{-t}(x)=e^{-i t x}\left(1+\mathcal{R}_{1}(t, x)\right), \quad\left|\mathcal{R}_{1}(t, x)\right| \leq c|t|^{-1}, \\
\sqrt{A(x)} \widetilde{\boldsymbol{\Phi}}_{-t}(x)=e^{-i t x}\left(1+\mathcal{R}_{2}(t, x)\right), \quad\left|\mathcal{R}_{2}(t, x)\right| \leq c|t|^{-1}, \\
c(t)^{-1}=\widetilde{\boldsymbol{c}}(t)^{-1}(1+E(t)), \quad|E(t)| \leq c|t|^{-1} .
\end{gathered}
$$

Therefore

$$
W_{R}^{\beta}(x, y)-V_{R}^{\beta}(x, y)=\frac{1}{2 \pi} \sqrt{A(y) / A(x)}\left(I_{1}+I_{2}+I_{3}\right),
$$

where

$$
\begin{aligned}
& I_{1}=\int_{\omega}\left(1-(t / R)^{2}\right)^{\beta} \sqrt{y^{2 \alpha+1}} \widetilde{\boldsymbol{\varphi}}_{t}(y) e^{-i t x}\left(\mathcal{R}_{1}(t, x)-\mathcal{R}_{2}(t, x)\right) \widetilde{\boldsymbol{c}}(t)^{-1} d t, \\
& I_{2}=\int_{\omega}\left(1-(t / R)^{2}\right)^{\beta} \sqrt{y^{2 \alpha+1}} \widetilde{\boldsymbol{\varphi}}_{t}(y) e^{-i t x}\left(1+\mathcal{R}_{1}(t, x)\right) \widetilde{\boldsymbol{c}}(t)^{-1} E(t) d t, \\
& I_{3}=\int_{\omega}\left(1-(t / R)^{2}\right)^{\beta} R_{0}(t, y) e^{-i t x}\left(1+\mathcal{R}_{1}(t, x)\right) \widetilde{\boldsymbol{c}}(t)^{-1}(1+E(t)) d t .
\end{aligned}
$$

The desired estimate now follows by taking absolute values inside the integral sign, along with well known estimates for Bessel functions,

$$
\left|\widetilde{\boldsymbol{\varphi}}_{t}(y)\right| \leq c(1+|t y|)^{-\alpha-1 / 2} e^{|\operatorname{Im} t| y}, \quad y>0, t \in \mathbb{C}-\{0\} .
$$


Let us show the case of $I_{1}$, the other two cases being similar:

$$
\begin{aligned}
\left|I_{1}\right| \leq & c\left(\frac{R y}{1+R y}\right)^{\alpha+1 / 2} \int_{0}^{\pi}\left|1-e^{-2 i \theta}\right|^{\beta} e^{-R(x-y) \sin \theta} d \theta \\
& \leq c\left(\frac{R y}{1+R y}\right)^{\alpha+1 / 2}(1+R(x-y))^{-\beta-1} .
\end{aligned}
$$

If, on the other hand, $0<x<y$, then switching variables,

$$
\begin{aligned}
& \left|W_{R}^{\beta}(x, y)-V_{R}^{\beta}(x, y)\right|=\frac{A(y)}{A(x)}\left|W_{R}^{\beta}(y, x)-V_{R}^{\beta}(y, x)\right| \\
& \leq c\left(\frac{R y}{1+R y}\right)^{\alpha+1 / 2} \sqrt{A(y) / A(x)}(1+R(y-x))^{-\beta-1} .
\end{aligned}
$$

In particular, for every $0<x<+\infty$ and $0<y<+\infty$,

$$
\left|V_{R}^{\beta}(x, y)-W_{R}^{\beta}(x, y)\right| \leq c\left(\frac{R y}{1+R y}\right)^{\alpha+1 / 2} \frac{\sqrt{A(y) / A(x)}}{(1+R|x-y|)^{\beta+1}} .
$$

It remains to show that if $\varepsilon<x<+\infty, 0<y<+\infty$, and $R$ is large, then

$$
\left(\frac{R y}{1+R y}\right)^{\alpha+1 / 2} \frac{\sqrt{A(y) / A(x)}}{(1+R|x-y|)^{\beta+1}} \leq c \frac{\sqrt{A(y)} y^{\lambda}}{(1+y)^{\lambda+\beta+1}} .
$$

This inequality is elementary. It suffices to consider separately the cases $0<y \leq$ $1 / R, 1 / R \leq y \leq x / 2, x / 2 \leq y \leq 2 x$, and $y \geq 2 x$. This proves (1), and (2) follows from the inversion formula for smooth rapidly decreasing functions.

Lemma 5. For every $\varepsilon<x<\eta$ and every function $f(x)$,

$$
\lim _{R \rightarrow+\infty}\left\{\left|V_{R}^{\beta} f(x)-T_{R}^{\beta} f(x)\right|\right\}=0 .
$$

ProOF. It just suffices to check that under the assumptions of Theorem 2, also Theorem 1 applies. Recall that

$$
V_{R}^{\beta} f(x)=\sqrt{x^{2 \alpha+1} / A(x)} S_{R}^{\beta}\left(\sqrt{A(y) / y^{2 \alpha+1}} f(y)\right)(x) .
$$

Then define

$$
U_{R}^{\beta} f(x)=\sqrt{x^{2 \alpha+1} / A(x)} T_{R}^{\beta}\left(\sqrt{A(y) / y^{2 \alpha+1}} f(y)\right)(x) .
$$

By Theorem $1, V_{R}^{\beta} f(x)$ and $U_{R}^{\beta} f(x)$ are equiconvergent. Moreover, as in the proof of Lemma 2, one can easily get rid of the factor $\sqrt{A(y) / y^{2 \alpha+1}}$. Hence also $U_{R}^{\beta} f(x)$ and $T_{R}^{\beta} f(x)$ are equiconvergent.

Corollary 2. For every $f(x)$ in $L^{p}\left(\mathbb{R}_{+}, A(x) d x\right), 1 \leq p \leq+\infty$, and every $\varepsilon<x<\eta$ the means $W_{R}^{\beta} f(x)$ and $T_{R}^{\beta} f(x)$ are equiconvergent under the following assumptions:

$$
\begin{aligned}
& \text { (1) } \rho=0 \text { and } \frac{2 b-2 \beta+1}{4 b+4}<\frac{1}{p}<\frac{2 \alpha+2 \beta+3}{4 \alpha+4} \text {, } \\
& \text { (2) } \rho>0 \text { and } \frac{1}{2} \leq \frac{1}{p}<\frac{2 \alpha+2 \beta+3}{4 \alpha+4} \text {. }
\end{aligned}
$$


Proof. This follows from Hölder's inequality. It suffices to recall that when $\rho=0$ then $A(x) \approx c x^{2 b+1}$ as $x \rightarrow+\infty$, while from the identity $\lim _{x \rightarrow+\infty} A^{\prime}(x) / A(x)=$ $2 \rho$ it follows that when $\rho>0$ then $c_{1} \exp ((2 \rho-\varepsilon) x) \leq A(x) \leq c_{2} \exp ((2 \rho+\varepsilon) x)$ as $x \rightarrow+\infty$, with $c_{1}$ and $c_{2}$ depending on $\varepsilon$.

\section{Bochner Riesz means in Sobolev spaces}

The fractional powers of the differential operator $\mathcal{L}=-A^{-1}(x) \frac{d}{d x}\left(A(x) \frac{d}{d x}\right)$ are defined spectrally for any complex $\gamma$ by

$$
(I+\mathcal{L})^{-\gamma / 2} g(x)=\int_{0}^{+\infty}\left(1+\rho^{2}+t^{2}\right)^{-\gamma / 2} \mathcal{F} g(t) \varphi_{t}(x) \frac{d t}{2 \pi|c(t)|^{2}} .
$$

The Sobolev spaces $\mathbb{W}^{\gamma, p}\left(\mathbb{R}_{+}, A(x) d x\right), \gamma \geq 0$ and $1 \leq p \leq+\infty$, are defined as the spaces of all distributions $f(x)=(I+\mathcal{L})^{-\gamma / 2} g(x)$, with $g(x)$ in $\mathbb{L}^{p}\left(\mathbb{R}_{+}, A(x) d x\right)$ and with norm $\|f\|_{\mathbb{W} \gamma, p}=\|g\|_{\mathbb{L}^{p}}$. In particular, $f(x)$ is in $\mathbb{W}^{2 n, p}\left(\mathbb{R}_{+}, A(x) d x\right)$ if and only if $\mathcal{L}^{j} f(x)$ is in $\mathbb{L}^{p}\left(\mathbb{R}_{+}, A(x) d x\right)$ for all $j=0,1, \ldots, n$. Another possible definition of $\mathbb{W}^{\gamma, p}\left(\mathbb{R}_{+}, A(x) d x\right), 0<\gamma<2 n$, is the complex interpolation space $\left[\mathbb{X}_{0}, \mathbb{X}_{n}\right]_{\gamma / 2 n}$, with $\mathbb{X}_{k}$ defined by the norm

$$
\|f\|_{\mathbb{X}_{k}}=\sum_{j=0}^{k}\left\{\int_{0}^{+\infty}\left|\mathcal{L}^{j} f(x)\right|^{p} A(x) d x\right\}^{1 / p} .
$$

In what follows we shall assume that these Sobolev spaces and fractional integral operators satisfy some classical imbedding properties:

(1) If $1=p<q<+\infty$ and $\gamma>(2 \alpha+2)(1 / p-1 / q)$, or if $1<p<q<+\infty$ and $\gamma \geq(2 \alpha+2)(1 / p-1 / q)$, then $(I+\mathcal{L})^{-\gamma / 2}$ is bounded from $\mathbb{L}^{p}\left(\mathbb{R}_{+}, A(x) d x\right)$ into $\mathbb{L}^{p}\left(\mathbb{R}_{+}, A(x) d x\right) \cap \mathbb{L}^{q}\left(\mathbb{R}_{+}, A(x) d x\right)$.

(2) If $1<p<+\infty$ and $-\infty<\tau<+\infty$, then the imaginary powers $(I+\mathcal{L})^{-i \tau / 2}$ are bounded on $\mathbb{L}^{p}\left(\mathbb{R}_{+}, A(x) d x\right)$, with norm of polynomial growth in $\tau$.

These properties are known to hold in a number of significant cases. They hold when $\lim _{x \rightarrow+\infty} A^{\prime}(x) / A(x)=2 \rho>0$, and when $\rho=0$ and $A(x)=x^{2 \alpha+1}$, and we suspect that they hold in general also when $\rho=0$. In particular, this covers the analysis of radial functions in Euclidean and non Euclidean spaces. The classical proof of (1) follows from Gaussian estimates for the heat kernel $\exp (-t \mathcal{L})$ and the subordination

$$
(I+\mathcal{L})^{-\gamma / 2}=\frac{1}{\Gamma(\gamma / 2)} \int_{0}^{+\infty} \exp (-t \mathcal{L}) \exp (-t) t^{\gamma / 2} \frac{d t}{t} .
$$

Moreover, (2) follows from the Hörmander multiplier theorem. For a proof in the Euclidean case see [25], and for Sturm Liouville expansions see [2].

The set of divergence of Bochner Riesz means is defined by

$$
D(\beta, f)=\left\{0 \leq x<+\infty, \lim _{R \rightarrow+\infty}\left\{W_{R}^{\beta} f(x)\right\} \text { does not exists }\right\} .
$$

THEOREM 3. Let $1 \leq p<+\infty, \beta \geq 0, \gamma \geq 0$, and assume that one of the following holds:

(1) $\rho=0$ and $\frac{2 b-2 \beta+1}{4 b+4}<\frac{1}{p}<\frac{2 \alpha+2 \beta+2 \gamma+3}{4 \alpha+4}$, 
(2) $\rho>0$ and $\frac{1}{2} \leq \frac{1}{p}<\frac{2 \alpha+2 \beta+2 \gamma+3}{4 \alpha+4}$. has:

Then, for every $f(x)=(I+\mathcal{L})^{-\gamma / 2} g(x)$, with $g(x)$ in $L^{p}\left(\mathbb{R}_{+}, A(x) d x\right)$, one

(A) If $0 \leq \gamma \leq 1 / p$, the divergence set of $W_{R}^{\beta} f(x)$ has Hausdorff dimension at most $1-\gamma p$.

(B) If $1 / p<\gamma \leq(2 \alpha+2) / p$, the divergence set either is empty or reduces to the origin.

(C) If $\gamma>(2 \alpha+2) / p$ and if $1 \leq p \leq 2$, the convergence holds everywhere.

Observe that when $p<2$ then the critical index for summability $\beta$ improves when the index of smoothness $\gamma$ increases, but when $p \geq 2$ then the smoothness does not lower the critical index. Also observe that the lower bound for the index $p$ depends on the local space dimension $2 \alpha+2$, while the upper bound depends on the space dimension $2 b+2$ at infinity.

Proof. The case $p=1$ and $\gamma=0$ in (1) for trigonometric expansions is the classical result of Bochner, and for Sturm Liouville expansions it follows from Corollary 2. The case $p=1$ and $\gamma>0$ follows from the case $1<p<+\infty$. Indeed a function with $\gamma$ derivatives in $\mathbb{L}^{1}\left(\mathbb{R}_{+}, A(x) d x\right)$ has $0 \leq \delta<\gamma$ derivatives in $\mathbb{L}^{p}\left(\mathbb{R}_{+}, A(x) d x\right)$ for every $p<(2 \alpha+2) /(2 \alpha+2+\delta-\gamma)$. When $p>1$ it suffices to prove that the divergence set $D(\beta, f) \cap(\varepsilon, \eta)$ has Hausdorff dimension at most $1-\gamma p$ for every $0<\varepsilon<\eta<+\infty$. In order to prove this, first we shall prove that, under the above assumptions, the $2 \alpha+2$ dimensional means $W_{R}^{\beta} f(x)$ are equiconvergent in $\varepsilon<x<\eta$ with the 1 dimensional means $T_{R}^{\beta} f(x)$, then we shall prove that functions with $\gamma$ derivatives in $\mathbb{L}^{p}\left(\mathbb{R}_{+}, A(x) d x\right)$ can be defined up to sets with Hausdorff dimension $1-\gamma p$, and that the divergence set of $T_{R}^{\beta} f(x)$ has dimension at most $1-\gamma p$. As before, in order to simplify the exposition, the proof is splitted into a series of lemmas.

LEMMA 6. Under the assumptions (1) and (2) of Theorem 3, if $f(x)=(I+\mathcal{L})^{-\gamma / 2} g(x)$ with $g(x)$ in $L^{p}\left(\mathbb{R}_{+}, A(x) d x\right)$, then the means $W_{R}^{\beta} f(x)$ and $T_{R}^{\beta} f(x)$ are equiconvergent in $\varepsilon<x<\eta$.

Proof. The case $\gamma=0$ is Corollary 2. The case $\gamma>0$ follows from the case $\gamma=0$ and the imbedding properties of the fractional integral operators.

In order to prove $(\mathrm{A})$ in Theorem 3, it then suffices to show that if $g(x)$ is in $\mathbb{L}^{p}\left(\mathbb{R}_{+}, A(x) d x\right)$, then $(I+\mathcal{L})^{-\gamma / 2} g(x)$ and $\lim _{R \rightarrow+\infty}\left\{T_{R}^{\beta}\left((I+\mathcal{L})^{-\gamma / 2} g\right)(x)\right\}$ exist up to sets with Hausdorff dimension at most $1-\gamma p$.

LEMMA 7. Let $\chi(x)$ be a smooth function with support in $0<\varepsilon / 3 \leq x \leq$ $3 \eta<+\infty$, and let $1<p<+\infty$ and $\gamma \geq 0$. Then for every function $g(x)$ in $L^{p}\left(\mathbb{R}_{+}, A(x) d x\right)$ there exists a function $h(x)$ in $L^{p}\left(\mathbb{R}_{+}, d x\right)$ such that

$$
\chi(x)(I+\mathcal{L})^{-\gamma / 2} g(x)=\left(I-d^{2} / d x^{2}\right)^{-\gamma / 2} h(x) .
$$

Proof. The meaning of the lemma is quite simple. On the support of $\chi(x)$ the measures $d x$ and $A(x) d x$ are comparable and on this support the associated Sobolev classes coincide. The details of the proof are more complicated. Denote by 
$\chi$ the operator of multiplication by $\chi(x)$. It suffices to prove the boundedness from $\mathbb{L}^{p}\left(\mathbb{R}_{+}, A(x) d x\right)$ into $\mathbb{L}^{p}\left(\mathbb{R}_{+}, d x\right)$ of the operator

$$
P^{\gamma} g(x)=\left(I-d^{2} / d x^{2}\right)^{\gamma / 2} \chi(I+\mathcal{L})^{-\gamma / 2} g(x) .
$$

First assume that $\gamma=2$. Observe that $\left(I-d^{2} / d x^{2}\right)=(I+\mathcal{L})+\mathcal{H}$ and $(I+\mathcal{L}) \chi=$ $\chi(I+\mathcal{L})+\mathcal{K}$, with $\mathcal{H}$ and $\mathcal{K}$ first order differential operators. Hence,

$$
\begin{gathered}
P^{2} g(x)=\left(I-d^{2} / d x^{2}\right) \chi(I+\mathcal{L})^{-1} g(x) \\
=(I+\mathcal{L}) \chi(I+\mathcal{L})^{-1} g(x)+\mathcal{H} \chi(I+\mathcal{L})^{-1} g(x) \\
=\chi g(x)+(\mathcal{K}+\mathcal{H} \chi)(I+\mathcal{L})^{-1} g(x) \\
=A(x)(I+\mathcal{L})^{-1} g(x)+B(x)(d / d x)(I+\mathcal{L})^{-1} g(x)+C(x) g(x) .
\end{gathered}
$$

Then assume that $\gamma=2 n$ is an even integer. By iterating the above argument one obtains that, for some smooth compactly supported functions $A_{j}(x)$ and $B_{j}(x)$,

$$
P^{2 n} g(x)=\sum_{j=0}^{n} A_{j}(x)(I+\mathcal{L})^{-j} g(x)+\sum_{j=1}^{n} B_{j}(x)(d / d x)(I+\mathcal{L})^{-j} g(x) .
$$

By our assumptions, the fractional integral operators $(I+\mathcal{L})^{-j}$ are bounded on $\mathbb{L}^{p}\left(\mathbb{R}_{+}, A(x) d x\right)$, hence the operators $A_{j}(x)(I+\mathcal{L})^{-j} g(x)$ are bounded from $\mathbb{L}^{p}\left(\mathbb{R}_{+}, A(x) d x\right)$ into $\mathbb{L}^{p}\left(\mathbb{R}_{+}, d x\right)$. In order to show that also the operators $B_{j}(x)(d / d x)(I+\mathcal{L})^{-j} g(x)$ are bounded, it suffices to show that the norm of $B_{j}(x)(d / d x)(I+\mathcal{L})^{-j} g(x)$ is controlled by the norms of $(I+\mathcal{L})^{-j} g(x)$ and $(I+\mathcal{L})^{1-j} g(x)$. More precisely, writing $(I+\mathcal{L})^{-j} g(x)=G(x)$, it suffices to show that

$$
\begin{gathered}
\left\{\int_{\varepsilon / 3}^{3 \eta}\left|\frac{d}{d x} G(x)\right|^{p} d x\right\}^{1 / p} \\
\leq c\left(\left\{\int_{\varepsilon / 3}^{3 \eta}|G(x)|^{p} d x\right\}^{1 / p}+\left\{\int_{\varepsilon / 3}^{3 \eta}\left|\left(1-\frac{A^{\prime}(x)}{A(x)} \frac{d}{d x}-\frac{d^{2}}{d x^{2}}\right) G(x)\right|^{p} d x\right\}^{1 / p}\right) .
\end{gathered}
$$

This follows from the classical inequality, see [17, Theorem 5.6, Chapter 2],

$$
\begin{gathered}
\left\{\int_{\varepsilon / 3}^{3 \eta}\left|\frac{d}{d x} G(x)\right|^{p} d x\right\}^{1 / p} \\
\leq c\left(t^{-1}\left\{\int_{\varepsilon / 3}^{3 \eta}|G(x)|^{p} d x\right\}^{1 / p}+t\left\{\int_{\varepsilon / 3}^{3 \eta}\left|\frac{d^{2}}{d x^{2}} G(x)\right|^{p} d x\right\}^{1 / p}\right) .
\end{gathered}
$$

Hence $P^{2 n}$ is a bounded operator from $\mathbb{L}^{p}\left(\mathbb{R}_{+}, A(x) d x\right)$ into $\mathbb{L}^{p}\left(\mathbb{R}_{+}, d x\right)$. Moreover, by the assumptions that $\left(I-d^{2} / d x^{2}\right)^{i \tau / 2}$ and $(I+\mathcal{L})^{-i \tau / 2}$ are bounded operators on $\mathbb{L}^{p}\left(\mathbb{R}_{+}, d x\right)$ and $\mathbb{L}^{p}\left(\mathbb{R}_{+}, A(x) d x\right)$ with norm of polynomial growth in $\tau$, it also follows that $P^{2 n+i \tau}=\left(I-d^{2} / d x^{2}\right)^{i \tau / 2} P^{2 n}(I+\mathcal{L})^{-i \tau / 2}$ is a bounded operator from $\mathbb{L}^{p}\left(\mathbb{R}_{+}, A(x) d x\right)$ into $\mathbb{L}^{p}\left(\mathbb{R}_{+}, d x\right)$, with norm of polynomial growth in $\tau$. Finally, the boundedness of $P^{\gamma}$ with $0<\gamma<2 n$ follows by complex interpolation between $0+i \tau$ and $2 n+i \tau$. 
Recall that the $(\gamma, p)$ capacity of a set $X \subseteq R_{+}, 0 \leq \gamma \leq 1 / p$ and $1<p<+\infty$, is defined by

$$
C(\gamma, p, X)=\inf \left\{\|h\|_{\mathbb{L}^{p}\left(\mathbb{R}_{+}, d x\right)}^{p},\left(I-d^{2} / d x^{2}\right)^{-\gamma / 2} h(x) \geq 1 \text { for every } x \text { in } X\right\} .
$$

LEMMA 8. If $g(x)$ is in $L^{p}\left(\mathbb{R}_{+}, A(x) d x\right)$, then

$$
(I+\mathcal{L})^{-\gamma / 2} g(x)
$$

and

$$
\lim _{R \rightarrow+\infty}\left\{T_{R}^{\beta}(I+\mathcal{L})^{-\gamma / 2} g(x)\right\}
$$

exist and are finite in sets whose complements have $(\gamma, p)$ capacity 0.

Proof. Observe that when $\gamma=0$, then the $(0, p)$ capacity coincide with Lebesgue measure. Also observe that for every $h(x)$ in $\mathbb{L}^{p}\left(\mathbb{R}_{+}, d x\right)$, then

$$
C\left(\gamma, p,\left\{\left|\left(I-d^{2} / d x^{2}\right)^{-\gamma / 2} h(x)\right| \geq t\right\}\right) \leq t^{-p}\|h\|_{\mathbb{L}^{p}\left(\mathbb{R}_{+}, d x\right)}^{p} .
$$

It then follows that $\left(I-d^{2} / d x^{2}\right)^{-\gamma / 2} h(x)$ can be infinite only in a set with $(\gamma, p)$ capacity 0 . Since, by Lemma $7, \chi(x)(I+\mathcal{L})^{-\gamma / 2} g(x)=\left(I-d^{2} / d x^{2}\right)^{-\gamma / 2} h(x)$, also $(I+\mathcal{L})^{-\gamma / 2} g(x)$ can be infinite only in a set with $(\gamma, p)$ capacity 0 . The existence of $\lim _{R \rightarrow+\infty}\left\{T_{R}^{\beta}(I+\mathcal{L})^{-\gamma / 2} g(x)\right\}$ follows from the boundedness of the associated maximal operator. Let $\mathcal{T}_{R}^{\beta}$ be the one dimensional Bochner Riesz operator, so that $T_{R}^{\beta} f(x)=\mathcal{T}_{R}^{\beta}(\chi f)(x)$ and $T_{R}^{\beta}(I+\mathcal{L})^{-\gamma / 2} g(x)=\mathcal{T}_{R}^{\beta}\left(I-d^{2} / d x^{2}\right)^{-\gamma / 2} h(x)$. Since the operators $\mathcal{T}_{R}^{\beta}$ and $\left(I-d^{2} / d x^{2}\right)^{-\gamma / 2}$ commute and since the fractional integral $\left(I-d^{2} / d x^{2}\right)^{-\gamma / 2}$ is a positive operator, one has

$$
\sup _{R>0}\left\{\left|\mathcal{T}_{R}^{\beta}\left(I-d^{2} / d x^{2}\right)^{-\gamma / 2} h(x)\right|\right\} \leq\left(I-d^{2} / d x^{2}\right)^{-\gamma / 2}\left(\sup _{R>0}\left\{\left|\mathcal{T}_{R}^{\beta} h\right|\right\}\right)(x) .
$$

Moreover,

$$
\left\{\int_{0}^{+\infty}\left|\sup _{R>0}\left\{\left|\mathcal{T}_{R}^{\beta} h(x)\right|\right\}\right|^{p} d x\right\}^{1 / p} \leq c\left\{\int_{0}^{+\infty}|h(x)|^{p} d x\right\}^{1 / p} .
$$

The boundedness of the maximal partial sum operator $\sup _{R>0}\left\{\left|\mathcal{T}_{R}^{\beta} h(x)\right|\right\}$ when $\beta=0$ and $1<p<+\infty$ comes from the Carleson Hunt theorem. The case $\beta>0$ is simpler. By Lemma 1 and the estimate $\left|J_{\beta+1 / 2}(z)\right| \leq c|z|^{-1 / 2}$,

$$
\left|\mathcal{T}_{R}^{\beta}(x, y)\right| \leq c R(1+R|x-y|)^{-\beta-1} .
$$

It then follows that when $\beta>0$, then the maximal operator $\sup _{R>0}\left\{\left|\mathcal{T}_{R}^{\beta} h(x)\right|\right\}$ is dominated by the Hardy Littlewood maximal operator, which is bounded on $\mathbb{L}^{p}\left(\mathbb{R}_{+}, d x\right)$. For every function $w(x)$ smooth with compact support in $\mathbb{R}_{+}$and every $x$,

$$
\lim _{R \rightarrow+\infty}\left\{\mathcal{T}_{R}^{\beta}\left(I-d^{2} / d x^{2}\right)^{-\gamma / 2} w(x)\right\}=\left(I-d^{2} / d x^{2}\right)^{-\gamma / 2} w(x) .
$$


Then, for every $\varepsilon>0$,

$$
\begin{gathered}
X(\varepsilon)= \\
\left\{x: \limsup _{R \rightarrow+\infty}\left\{\left|\mathcal{T}_{R}^{\beta}\left(I-d^{2} / d x^{2}\right)^{-\gamma / 2} h(x)-\left(I-d^{2} / d x^{2}\right)^{-\gamma / 2} h(x)\right|\right\}>\varepsilon\right\} \\
\subseteq\left\{x:\left(I-d^{2} / d x^{2}\right)^{-\gamma / 2} \sup _{R>0}\left\{\left|\mathcal{T}_{R}^{\beta}(h-w)(x)\right|\right\}>\varepsilon / 2\right\} \\
\cup\left\{x:\left(I-d^{2} / d x^{2}\right)^{-\gamma / 2}|h-w|(x)>\varepsilon / 2\right\} .
\end{gathered}
$$

Hence,

$$
C(\gamma, p, X(\varepsilon)) \leq c \varepsilon^{-p}\left\{\int_{0}^{+\infty}|h(x)-w(x)|^{p} d x\right\}^{1 / p} .
$$

Since $\int_{0}^{+\infty}|h(x)-w(x)|^{p} d x$ can be chosen arbitrarily small, $C(\gamma, p, X(\varepsilon))=0$. Finally, since capacity is subadditive, also $C\left(\gamma, p, \cup_{\varepsilon>0} X(\varepsilon)\right)=0$.

In order to conclude the proof of $(\mathrm{A})$, it suffices to recall that sets with $(\gamma, p)$ capacity 0 have Hausdorff dimension at most $1-\gamma p$. See e.g. [29]. (B) in Theorem 3 follows from Corollary 2 and the following lemma.

Lemma 9. If $g(x)$ is in $L^{p}\left(\mathbb{R}_{+}, A(x) d x\right)$ and if $\gamma>1 / p$, then $\chi(x)(I+\mathcal{L})^{-\gamma / 2} g(x)$ is bounded and Hölder continuous of order $\gamma-1 / p$, and

$$
\lim _{R \rightarrow+\infty}\left\{T_{R}^{\beta}(I+\mathcal{L})^{-\gamma / 2} g(x)\right\}=f(x) .
$$

Proof. By Lemma $7, \chi(x)(I+\mathcal{L})^{-\gamma / 2} g(x)=\left(I-d^{2} / d x^{2}\right)^{-\gamma / 2} h(x)$ with $h(x)$ is in $\mathbb{L}^{p}\left(\mathbb{R}_{+}, d x\right)$. By the Sobolev imbedding theorem, functions with $\gamma$ derivatives in $\mathbb{L}^{p}\left(\mathbb{R}_{+}, d x\right)$ are Hölder continuous of order $\gamma-1 / p$. Finally, the pointwise convergence of $T_{R}^{\beta}(I+\mathcal{L})^{-\gamma / 2} g(x)$ follows from the Dini criterion for convergence of Fourier series and integrals.

Finally, in order to prove (C) in Theorem 3 it suffices to prove that the linear functionals which associate to a function $g(x)$ in $\mathbb{L}^{p}\left(\mathbb{R}_{+}, A(x) d x\right)$ the numbers $W_{R}^{\beta}(I+\mathcal{L})^{-\gamma / 2} g(0)$ are uniformly bounded. This follows from the following lemma.

LEMMA 10. Let

$$
\begin{gathered}
I^{\gamma}(x)=\int_{0}^{+\infty}\left(1+\rho^{2}+t^{2}\right)^{-\gamma / 2} \varphi_{t}(x) \frac{d t}{2 \pi|c(t)|^{2}} \\
W_{R}^{\beta} I^{\gamma}(x)=\int_{0}^{R}\left(1-(t / R)^{2}\right)^{\beta}\left(1+\rho^{2}+t^{2}\right)^{-\gamma / 2} \varphi_{t}(x) \frac{d t}{2 \pi|c(t)|^{2}} .
\end{gathered}
$$

Then, under the assumptions (1) or (2) of Theorem 3, if $\gamma>(2 \alpha+2) / p$ and if $1 \leq p \leq 2$ and $1 / p+1 / q=1$,

$$
\sup _{R>0}\left\{\int_{0}^{+\infty}\left|W_{R}^{\beta} I^{\gamma}(x)\right|^{q} A(x) d x\right\}^{1 / q}<+\infty .
$$


ProOF. The result follows by the Hausdorff Young inequality,

$$
\begin{gathered}
\left\{\int_{0}^{+\infty}\left|W_{R}^{\beta} I^{\gamma}(x)\right|^{q} A(x) d x\right\}^{1 / q} \\
\leq\left\{\int_{0}^{R}\left|\left(1-(t / R)^{2}\right)^{\beta}\left(1+\rho^{2}+t^{2}\right)^{-\gamma / 2}\right|^{p} \frac{d t}{2 \pi|c(t)|^{2}}\right\}^{1 / p} \\
\leq\left\{\int_{0}^{+\infty}\left|\left(1+\rho^{2}+t^{2}\right)^{-\gamma / 2}\right|^{p} \frac{d t}{2 \pi|c(t)|^{2}}\right\}^{1 / p}<+\infty .
\end{gathered}
$$

As in the classical case, this Hausdorff Young inequality follows by interpolation between $(1,+\infty)$ and $(2,2)$.

The following remarks show that the ranges of indexes in the above theorem are best possible, with a possible exception of (C) with $\rho=0$ and $2<p<$ $(4 b+4) /(2 b-2 \beta+1)$.

REMARK 2. The upper value for the dimension of divergence sets in Theorem 3 is optimal. Indeed, functions with $\gamma \leq(2 \alpha+2) / p$ derivatives in $\mathbb{L}^{p}\left(\mathbb{R}_{+}, A(x) d x\right)$ may be unbounded at the origin. This follows from the non integrability of the Fourier transform of such functions. Moreover, if $\gamma<1 / p$ then they may be infinite on sets with Hausdorff dimension $1-\gamma p$. In order to see this, observe that, by Lemma 7 , it suffices to prove this when $A(x)=1$ and with $\mathbb{R}$ instead of $\mathbb{R}_{+}$. Next observe that a set $X$ has $(\gamma, p)$ capacity 0 if and only if there exists a function $h(x)$ in $\mathbb{L}^{p}\left(\mathbb{R}_{+}, d x\right)$ with $\left(I-d^{2} / d x^{2}\right)^{-\gamma / 2} h(x)=+\infty$ for every $x$ in $X$. Finally recall that sets with $(\gamma, p)$ capacity 0 have $1-\gamma p+\varepsilon$ Hausdorff measure 0 for every $\varepsilon>0$. See e.g. [29, Theorem 2.6.16.]. For completeness we present a construction of such functions. For simplicity, we use the Riesz capacity instead of the Bessel one, but sets of Bessel or Riesz capacity zero are the same. Let $X$ be a compact set in $\mathbb{R}$ with positive and finite $\delta$ dimensional Hausdorff measure and let $d \mu(x)$ be the $\delta$ dimensional Hausdorff measure restricted to $X$, so that

$$
\begin{gathered}
\mu\{|x-y|<t\} \leq t^{\delta} \quad \text { for every } x \in \mathbb{R} \text { and } t>0, \\
\limsup _{t \rightsquigarrow 0+}\left\{\frac{\mu\{|x-y|<t\}}{t^{\delta}}\right\}>0 \quad \text { for } \mu \text { almost every } x \in X .
\end{gathered}
$$

For every $0<\varepsilon<1$, the Riesz potential

$$
R^{\varepsilon} * \mu(x)=\int_{X}|x-y|^{\varepsilon-1} d \mu(y)
$$

is locally integrable. This follows from the local integrability of $|x-y|^{\varepsilon-1}$. Moreover, if $\varepsilon>1-\delta$ then $R^{\varepsilon} * \mu(x)$ is uniformly bounded. This follows from the estimate $\mu\{|x-y|<t\} \leq t^{\delta}$. By Hölder's inequality, if $\varepsilon+(1-\delta)(1-1 / p)<1$, then

$$
\begin{gathered}
R^{\varepsilon+(1-\delta)(1-1 / p)} * \mu(x)=\int_{X}|x-y|^{\varepsilon+(1-\delta)(1-1 / p)-1} d \mu(y) \\
\leq\left\{\int_{X}|x-y|^{\varepsilon+(1-\delta)-1} d \mu(y)\right\}^{1-1 / p}\left\{\int_{X}|x-y|^{\varepsilon-1} d \mu(y)\right\}^{1 / p} .
\end{gathered}
$$

The first factor is uniformly bounded and the p power of the second factor is locally integrable, hence $R^{\varepsilon+(1-\delta)(1-1 / p)} * \mu(x)$ is locally in $\mathbb{L}^{p}(\mathbb{R}, d x)$. Finally, if $\gamma+\varepsilon+$ 
$(1-\delta)(1-1 / p)<1$ then

$$
R^{\gamma} * R^{\varepsilon+(1-\delta)(1-1 / p)} * \mu(x)=c R^{\gamma+\varepsilon+(1-\delta)(1-1 / p)} * \mu(x)
$$

is locally in $\mathbb{W}^{\gamma, p}(\mathbb{R}, d x)$. On the other hand,

$$
\begin{gathered}
R^{\gamma+\varepsilon+(1-\delta)(1-1 / p)} * \mu(x)=\int_{X}|x-y|^{\gamma+\varepsilon+(1-\delta)(1-1 / p)-1} d \mu(y) \\
\geq \limsup _{t \rightsquigarrow 0+}\left\{t^{\gamma+\varepsilon+(1-\delta)(1-1 / p)-1} \mu\{|x-y|<t\}\right\} \\
=\limsup _{t \rightsquigarrow 0+}\left\{t^{(\delta-1+\gamma p+\varepsilon p) / p} \frac{\mu\{|x-y|<t\}}{t^{\delta}}\right\} .
\end{gathered}
$$

If $\delta<1-\gamma p$, and if $\varepsilon$ is so small that $\delta-1+\gamma p+\varepsilon p<0$, then $R^{\gamma+\varepsilon+(1-\delta)(1-1 / p)} *$ $\mu(x)=+\infty$ for $\mu$ almost every $x$ in $X$.

REMARK 3. The function $I^{\delta}(x)$ with Fourier Bessel transform $\left(1+t^{2}\right)^{-\delta / 2}$ shows that the indexes for Bochner Riesz summability in Theorem 3 are best possible when $p<2$. This function is positive and smooth away from the origin, $I^{\delta}(x) \approx$ $c|x|^{\delta-2 \alpha-2}$ when $x \rightarrow 0$, and it has an exponential decay at infinity. See [25, Chapter V.3]. It then follows that this function is in $\mathbb{W}^{\gamma, p}\left(\mathbb{R}_{+}, x^{2 \alpha+1} d x\right)$ provided that $\gamma<\delta-(2 \alpha+2)(1-1 / p)$. Let $\varphi(t)$ be a smooth function with $\varphi(t)=1$ if $t \leq 1 / 3$ and $\varphi(t)=0$ if $t \geq 2 / 3$. Then

$$
\begin{gathered}
S_{R}^{\beta} I^{\delta}(x) \\
=\int_{0}^{2 R / 3} \varphi(t / R)\left(1-(t / R)^{2}\right)^{\beta}\left(1+t^{2}\right)^{-\delta / 2} \frac{J_{\alpha}(t x)}{(t x)^{\alpha}} t^{2 \alpha+1} d t \\
+\int_{R / 3}^{R}(1-\varphi(t / R))\left(1-(t / R)^{2}\right)^{\beta}\left(1+t^{2}\right)^{-\delta / 2} \frac{J_{\alpha}(t x)}{(t x)^{\alpha}} t^{2 \alpha+1} d t .
\end{gathered}
$$

The multiplier $\varphi(t)\left(1-t^{2}\right)^{\beta}$ is smooth and for almost every $x$,

$$
\lim _{R \rightarrow+\infty}\left\{\int_{0}^{2 R / 3} \varphi(t / R)\left(1-(t / R)^{2}\right)^{\beta}\left(1+t^{2}\right)^{-\delta / 2} \frac{J_{\alpha}(t x)}{(t x)^{\alpha}} t^{2 \alpha+1} d t\right\}=I^{\delta}(x) .
$$

Moreover, by the asymptotic expansion of the Bessel functions, for an appropriate function $\Phi(t)$, phases $\zeta$ and $\vartheta$, one has

$$
\begin{gathered}
\int_{R / 3}^{R}(1-\varphi(t / R))\left(1-(t / R)^{2}\right)^{\beta}\left(1+t^{2}\right)^{-\delta / 2} \frac{J_{\alpha}(t x)}{(t x)^{\alpha}} t^{2 \alpha+1} d t \\
\approx R^{\alpha-\delta+3 / 2} x^{-\alpha-1 / 2} \int_{1 / 3}^{1} \Phi(t)(1-t)^{\beta} \cos (R x t-\zeta) d t \\
\approx R^{\alpha-\beta-\delta+1 / 2} x^{-\alpha-\beta-3 / 2} \cos (R x-\vartheta) .
\end{gathered}
$$

In particular, if $\alpha-\beta-\delta+1 / 2 \geq 0$ this term does not converge when $R \rightarrow$ $\infty$. Hence a necessary condition for convergence is $\beta>\alpha-\delta+1 / 2$. Finally, since $\delta>\gamma+(2 \alpha+2)(1-1 / p)$, a necessary condition for convergence is $\beta+\gamma \geq$ $(2 \alpha+2)(1 / p-1 / 2)-1 / 2$.

REMARK 4. When $p>2$ an argument of Rubio de Francia shows that Bochner Riesz means of index $\beta \leq(2 \alpha+2)(1 / 2-1 / p)-1 / 2$ of functions in $\mathbb{W}^{\gamma, p}\left(\mathbb{R}_{+}, x^{2 \alpha+1} d x\right)$ are not tempered distributions. Suppose the contrary. Then by duality $S_{R}^{\beta}$ is also 
bounded from the space of test functions into $\mathbb{W}^{-\gamma, q}\left(\mathbb{R}_{+}, x^{2 \alpha+1} d x\right)$ with $1 / p+$ $1 / q=1$, and $(I+\mathcal{L})^{-\gamma / 2} S_{R}^{\beta}$ is bounded from the space of test functions into $\mathbb{L}^{q}\left(\mathbb{R}_{+}, x^{2 \alpha+1} d x\right)$. On the other hand, if $f(x)$ is a test function with $\mathcal{F}_{\alpha} f(t)=$ $\left(1+t^{2}\right)^{\gamma / 2}$ for all $t \leq R$, then

$$
(I+\mathcal{L})^{-\gamma / 2} S_{R}^{\beta} f(x)=\int_{0}^{R}\left(1-(t / R)^{2}\right)^{\beta} \frac{J_{\alpha}(t x)}{(t x)^{\alpha}} t^{2 \alpha+1} d t=c S_{R}^{\beta}(x, 0) .
$$

If $\beta \leq(2 \alpha+2)(1 / p-1 / 2)-1 / 2$ then this function is not in $\mathbb{L}^{q}\left(\mathbb{R}_{+}, x^{2 \alpha+1} d x\right)$. Indeed, by Lemma 2 (1), as $|x| \rightarrow+\infty$,

$$
\left|S_{R}^{\beta}(x, 0)\right| \approx c R^{\alpha-\beta+1 / 2}|x|^{-\alpha-\beta-3 / 2} .
$$

REMARK 5. For Fourier Bessel expansions the full range of indexes $p$ in Lemma 10, hence in part $(C)$ of Theorem 3, is $1 \leq p<(4 \alpha+4) /(2 \alpha-2 \beta+1)$. The case $1 \leq p \leq 2$ is already in the lemma. When $(2 \alpha-2 \beta+1) /(4 \alpha+4)<1 / p \leq 1 / 2$ and $\beta \geq 0$, then the operators $S_{R}^{\beta}$ are uniformly bounded on $\mathbb{L}^{q}\left(\mathbb{R}_{+}, x^{2 \alpha+1} d x\right)$. See $[\mathbf{2 2}, \mathbf{2 3}]$ for the case $\beta=0$, and $[\mathbf{1 5}, \mathbf{1 8}]$ for the case $\beta>0$. Moreover, the kernel $I^{\gamma}(x)$ is asymptotic to $c x^{\gamma-2 \alpha-2}$ at the origin with an exponential decay at infinity. Hence, if $\gamma>(2 \alpha+2) / p$ then $I^{\gamma}(x)$ is in $\mathbb{L}^{q}\left(\mathbb{R}_{+}, x^{2 \alpha+1} d x\right)$ and

$$
\left\{\int_{0}^{+\infty}\left|S_{R}^{\beta} I^{\gamma}(x)\right|^{q} x^{2 \alpha+1} d x\right\}^{1 / q} \leq c\left\{\int_{0}^{+\infty}\left|I^{\gamma}(x)\right|^{q} x^{2 \alpha+1} d x\right\}^{1 / q}<+\infty .
$$

REMARK 6. Bochner Riesz means with negative index in Sobolev spaces have been considered in $[\mathbf{3}]$.

\section{References}

[1] J.A.Barceló, J.Bennett, A.Carbery, K.M.Rogers: On the dimension of divergence sets of dispersive equations, Mathematische Annalen 349 (2011), 599-622.

[2] W.R.Bloom, Z.Xu: Fourier multipliers for $\mathbb{L}^{p}$ on Chébli-Trimèche hypergroups, Proceedings of the London Mathematical Society 80 (2000), 643-664.

[3] L.Brandolini, L.Colzani: Bochner-Riesz means with negative index of radial functions in Sobolev spaces, Rendiconti del Circolo Matematico di Palermo 42 (1993), 117-128.

[4] L.Brandolini, G.Gigante: Equiconvergence theorems for Chébli-Trimèche hypergroups, Annali Scuola Normale Superire Pisa Cl. Sci. 8 (2009), 211-265.

[5] A.Carbery: The boundedness of the maximal Bochner Riesz operator on $\mathbb{L}^{4}\left(\mathbb{R}^{2}\right)$, Duke Mathematical Journal 50 (1983), 409-416.

[6] A.Carbery, J.L.Rubio de Francia, L.Vega: Almost everywhere summability of Fourier integrals, Journal of the London Mathematical Society 38 (1988), 513-524.

[7] A.Carbery, F.Soria: Almost-everywhere convergence of Fourier integrals for functions in Sobolev spaces and an $L^{2}$ localisation principle, Revista Matemática Iberoamericana 4 (1988), 319-337.

[8] A.Carbery, F.Soria: Sets of divergence for the localization problem for Fourier integrals, Comptes Rendus de l'Académie des Sciences Série I Mathématique 325 (1997), no. 12, 12831286.

[9] A.Carbery, F.Soria: Pointwise Fourier inversion and localisation in $\mathbb{R}^{n}$, The Journal of Fourier Analysis and Applications 3 (1997), 847-858.

[10] A.Carbery, F.Soria, A.Vargas: Localisation and weighted inequalities for spherical Fourier means, Journal d'Analyse Mathématique 103 (2007), 133-156.

[11] M.J.Carro, E.Prestini: Convergence a.e. of spherical partial Fourier integrals on weighted spaces for radial functions: endpoint estimates, Studia Mathematica 192 (2009), 173-194.

[12] M.Christ: On almost everywhere convergence of Bochner Riesz means in higher dimensions, Proceedings of the American Mathematical Society 95 (1985), 16-20. 
[13] L.Colzani: Fourier expansions of functions of bounded variation of several variables, Transactions of the American Mathematical Society 358 (2006), 5501-5521.

[14] L.Colzani, A.Crespi, G.Travaglini, M.Vignati: Equiconvergence theorems for Fourier Bessel expansions with applications to the harmonic analysis of radial functions in Euclidean and non-Euclidean spaces, Transactions of the American Mathematical Society 338 (1993), 43-55.

[15] L.Colzani, G.Travaglini, M.Vignati: Bochner Riesz means of functions in weak-L $(p)$, Monatshefte fur Mathematik 115 (1993), 35-45.

[16] L.Colzani, S.Volpi: Pointwise convergence of Bochner-Riesz means in Sobolev spaces, preprint.

[17] R.A.DeVore, G.G.Lorentz: Constructive approximation, Spinger Verlag (1993).

[18] Y.Kanjin: Convergence almost everywhere of Bochner Riesz means for radial tions, Annals of Science Kanazawa University 25 (1988), 11-15.

[19] B.Ma: Almost everywhere convergence of Bochner-Riesz means in Bessel potential spaces, Approximation Theory and its Applications 13 (1997), 13-18.

[20] C.Meaney, E.Prestini: Bochner Riesz means on symmetric spaces, The Tohoku Mathematical Journal (2) $\mathbf{5 0}$ (1998), 557-570.

[21] E.Montini: On the capacity of sets of divergence associated with the spherical partial integrals operator, Transactions of the American Mathematical Society 355 (2002), 1415-1441.

[22] E.Prestini: Almost everywhere convergence for the spherical partial sums for radial functions, Monatshefte fur Mathematik 105 (1988), 207-216.

[23] E.Romera, F.Soria: Endpoint estimates for the maximal operator associated to spherical partial sums on radial functions, Proceedings of the American Mathematical Society 111 (1991), 1015-1022.

[24] J.L.Rubio de Francia: Transference principles for radial multipliers, Duke Mathematical Journal 58 (1989), 1-19.

[25] E.M.Stein: Singular integrals and differentiability properties of functions, Princeton University Press (1971).

[26] E.M.Stein, G.Weiss: Introduction to Fourier analysis on Euclidean spaces, Princeton University Press (1971).

[27] T.Tao: On the maximal Bochner Riesz conjecture in the plane for $p<2$, Transactions of the American Mathematical Society 354 (2002), 1947-1959.

[28] S.Volpi: Bochner Riesz means of eigenfunction expansions and local Hardy spaces on manifolds with bounded geometry, Ph.D. dissertation, Università di Milano Bicocca, 2012.

[29] W.P.Ziemer: Weakly differentiable functions, Sobolev spaces and functions of bounded variation, Springer Verlag (1989).

[30] A.Zygmund: Trigonometric series I, II, Cambridge University Press (1977).

(L. Colzani) Dipartimento di Matematica e Applicazioni, Edificio U5, Università di Milano Bicocca, Via R.Cozzi 53, 20125 Milano, Italia.

E-mail address: leonardo.colzani@unimib.it

(G. Gigante) Dipartimento di Ingegneria, Università di Bergamo, Viale Marconi 5 , 24044 Dalmine, Bergamo, Italia.

E-mail address: giacomo.gigante@unibg.it

(S. Volpi) Dipartimento di Matematica e Applicazioni, Edificio U5, Università di Milano Bicocca, Via R.Cozzi 53, 20125 Milano, Italia.

E-mail address: sara_volpi@yahoo.it 